\title{
Metadynamics study of the temperature dependence of magnetic anisotropy and spin-reorientation transitions in ultrathin films
}

\author{
Balázs Nagyfalusi $\odot,{ }^{1, *}$ László Udvardi ${ }^{1,2}$ and László Szunyogh $\oplus^{1,2}$ \\ ${ }^{1}$ Department of Theoretical Physics, Budapest University of Technology and Economics, Budafoki út 8, H-1111 Budapest, Hungary \\ ${ }^{2}$ MTA-BME Condensed Matter Research Group, Budapest University of Technology and Economics, \\ Budafoki út 8, H-1111 Budapest, Hungary
}

(Received 9 July 2019; revised manuscript received 9 October 2019; published 25 November 2019)

\begin{abstract}
We employ metadynamics simulations to calculate the free-energy landscape of thin ferromagnetic films and perform a systematic study of the temperature dependence of magnetic anisotropy and of the spinreorientation transitions. By using a simple spin model we recover the well-known power-law behavior of the magnetic anisotropy energy against magnetization and present a rather detailed analysis of the spin-reorientation transitions in ultrathin films. Based on tensorial exchange interactions and anisotropy parameters derived from first-principles calculations, we perform simulations for Fe double layers deposited on $\mathrm{Au}(001)$ and $\mathrm{W}(110)$. In the case of $\mathrm{Fe}_{2} \mathrm{~W}$ (110) our simulations display an out-of-plane to in-plane spin-reorientation transition in agreement with experiments.
\end{abstract}

DOI: 10.1103/PhysRevB.100.174429

\section{INTRODUCTION}

Since Néel's seminal paper in 1954 [1], considerable interest has been focused on the magnetism of thin films and multilayers. Magnetic anisotropy plays a key role in several phenomena important for technological applications. In a magnetic data storage device the information is stored by controlling the magnetic orientation of a small magnetic domain that is retained by magnetic anisotropy. In the early implementation of magnetic recording the magnetization of the bits was parallel with the plane of the film. Application of materials with perpendicular magnetic anisotropy (PMA) triggered an order of magnitude increase of the storage density. The first realization of a perpendicular magnetic recording occurred more than a decade ago [2]; recent reviews on PMA can be found in Refs. [3,4]. In order to further increase the storage density, the grain size in the recording medium should be decreased, which requires a high uniaxial magnetic anisotropy of the thin film. Due to the large magnetic anisotropy of the recording media the field produced by the write head might no longer be sufficient to overcome the barrier to switch the magnetization. To circumvent this issue a heat-assisted magnetic recording (HAMR) was proposed [5-7]. In HAMR the magnetic anisotropy is decreased by temporarily heating the domain storing the information.

The temperature dependence of magnetic anisotropy of thin films has been investigated both experimentally [8-11] and theoretically [12-15]. The magnetic anisotropy energy (MAE) at finite temperature is usually defined as the difference between the free energy of the in-plane magnetized system and that of the normal-to-plane magnetized system. Magnetic simulations provide different tools for sampling the complex free-energy surfaces. One branch of such schemes is formed by the adaptive biasing potential methods such as the Wang-Landau algorithm [16], umbrella sampling [17], and metadynamics [18]. In metadynamics a biasing potential is constructed as a sum of Gaussians centered along the trajectory in the space of the collective variables [18]. In well-tempered metadynamics the smooth convergence of the biasing potential is guaranteed by changing adaptively the height of the Gaussians [19]. This algorithm is proved to converge to the exact free energy [20]. Implemented in micromagnetic simulations, metadynamics has recently been employed to study the vortex nucleation process in a magnetic nanodot [21].

In this work we perform a systematic study of the temperature dependence of magnetic anisotropy and spinreorientation transitions (SRTs) by using metadynamics. In Sec. II we outline the main features of metadynamics simulations with the aim of studying the free-energy landscape of a thin ferromagnetic film. In Sec. III we first present a model study of the temperature dependence of magnetic anisotropy and a rather detailed analysis of the SRT in ultrathin films. Based on tensorial exchange interactions and anisotropy parameters derived from first-principles calculations, we then present simulations on Fe bilayers deposited on $\mathrm{Au}(001)$ and $\mathrm{W}(110)$, and finally, we summarize our results.

\section{DETAILS OF THE METADYNAMICS SIMULATIONS}

The magnetic properties of thin films of transition metals are often described by classical spin models [22]. In most of this work we choose a simple Heisenberg model to describe the magnetic properties of an ultrathin film with uniaxial anisotropy and anisotropic exchange interactions:

\footnotetext{
*nagyfalusi@phy.bme.hu
}

$$
H=-\frac{1}{2} \sum_{\langle i, j\rangle}\left(J \mathbf{s}_{i} \mathbf{s}_{j}-d s_{z i} s_{z j}\right)-\sum_{i} \lambda_{i} s_{z i}^{2},
$$


where $\mathbf{s}_{i}$ is a unit vector representing the direction of the atomic magnetic moment at site $i$, only nearest neighbors are considered in the first sum on the right-hand side with isotropic exchange coupling $J$ and an anisotropic part $d$, and $\lambda_{i}$ are the uniaxial anisotropy constants. More complex spin models will be presented and used only in Secs. III C and III $\mathrm{D}$ in the context of Fe bilayers on $\mathrm{Au}(001)$ and W(110).

The free energy is sampled along an appropriately chosen collective variable (CV) labeled by $\eta$. For our present study we chose the $z$ (normal-to-plane) component of the normalized magnetization, $\eta=M_{z} / M$, as the collective variable, where $M_{z}=\sum_{i} s_{z i}$ and $M=\left|\sum_{i} \mathbf{s}_{i}\right|$. The key quantity in metadynamics is the bias potential $V_{\mathrm{b}}(\eta)$ added to the energy of the system. Although in most of its applications metadynamics is implemented in molecular dynamics, there are examples where it is successfully used in Monte Carlo simulations $[23,24]$ as well. If a Monte Carlo step (MCS) is interpreted as a time step, the bias potential will be time dependent as well. After every $\tau$ MCS a Gaussian potential centered at the actual value of the $\mathrm{CV}, \eta_{\mathrm{act}}$, is added to the bias potential:

$$
\begin{gathered}
V_{\mathrm{b}}(\eta, t+\tau)=V_{\mathrm{b}}(\eta, t)+V_{\mathrm{G}}\left(\eta-\eta_{\mathrm{act}}\right), \\
V_{\mathrm{G}}\left(\eta-\eta_{\mathrm{act}}\right)=w e^{-\frac{\left(\eta-\eta_{\mathrm{act}}\right)^{2}}{2 \sigma}},
\end{gathered}
$$

where $\sigma$ and $w$ are the width and the height of the Gaussian, respectively. In well-tempered metadynamics [19] the height of the Gaussian is chosen to change with the time $\tau$.

In our metadynamics simulations we applied a simple Metropolis algorithm [25] with the probability of a random change in the spin at site $i \mathbf{s}_{i} \rightarrow \mathbf{s}_{i}^{\prime}$,

$$
P\left(\mathbf{s}_{i} \rightarrow \mathbf{s}_{i}^{\prime}\right)=\min \left\{1, e^{-\beta\left[E\left(\mathbf{s}^{\prime}\right)+V_{\mathrm{b}}\left(\eta\left(\mathbf{s}^{\prime}\right)\right)-E(\mathbf{s})-V_{\mathrm{b}}(\eta(\mathbf{s}))\right]}\right\},
$$

where $\beta$ is the inverse temperature and $E(\mathbf{s})$ is the energy of the spin configuration given by Eq. (1). After a predefined number of Monte Carlo steps the biasing potential is updated by adding a Gaussian centered at the actual value of the $\mathrm{CV}$ with a height of $w=w_{0} e^{-\frac{V_{\mathrm{b}}\left(\eta_{\mathrm{B}}\right.}{k_{\mathrm{B}} T_{\mathrm{m}}}}$, where $T_{\mathrm{m}}$ is an appropriately chosen temperature as is explained in the procedure of welltempered metadynamics [19]. In equilibrium, i.e., when the bias potential becomes stationary, the free energy $F(T)$ of the system is identified with the negative of the bias potential, $F(T)=-V_{\mathrm{b}}(\eta(T))$, where $\eta(T)$ stands for the equilibrium value of the CV [20].

The values of the $\mathrm{CV}$ chosen for our model must be within the interval $[-1,1]$, and the free energy has a discontinuity at the boundaries which cannot be accurately reproduced by a sum of finite-width Gaussians, as detailed in Refs. [24,26]. In order to eliminate this problem, the procedure proposed by Crespo et al. [24] has been modified in the following manner. Whenever the bias potential is updated, an extra Gaussian with the same width and height is added out of the physically relevant interval of the $\mathrm{CV}$ :

$$
\begin{aligned}
V_{\mathrm{b}}(\eta, t+\tau)= & V_{\mathrm{b}}(\eta, t)+V_{\mathrm{G}}\left(\eta-\eta_{\mathrm{act}}\right) \\
& + \begin{cases}V_{\mathrm{G}}\left(\eta-2+\eta_{\text {act }}\right) & \text { if } \eta>0, \\
V_{\mathrm{G}}\left(\eta+2+\eta_{\text {act }}\right) & \text { if } \eta<0,\end{cases}
\end{aligned}
$$

where $V_{\mathrm{G}}(\eta)$ is the Gaussian potential given by Eq. (3). This scheme clearly makes the bias potential continuous at $\eta=$ \pm 1 . It should be noted that $V_{\mathrm{b}}(\eta)$ does not go smoothly to zero in the nonphysical region, but this part of the $\mathrm{CV}$ is never sampled during the simulation. In order to explore the freeenergy surface along the CV multiple-walker metadynamics [27] was applied. The simulations were done simultaneously on typically four replicas, each contributing equally to the growth of a joint bias potential.

The parameters of the bias potential were optimized for each system under consideration. The half width $\sigma$ determines the grid on the space of the $\mathrm{CV}$, where the bias potential is sampled. In general, we chose $\sigma=0.03$, making it sufficient to sample 150 points in the $[-1: 1]$ interval of the $\mathrm{CV}$ in order to get a smooth curve for the free energy. The value of the metatemperature $T_{m}$ depends on the Curie temperature. Obviously, in the high- $T_{m}$ limit the normal (nontempered) metadynamics is regained, and the bias potential will not converge. If $T_{m}$ is too small, then the convergence will be very slow. In the present study a few times the Curie temperature is used for $T_{m}$. The parameter $w_{0}$ should be chosen to be considerably smaller than the anisotropy energy of the whole lattice.

Since the free energy is sampled along a simple onedimensional collective variable, it is worth mentioning that umbrella sampling [28] could also efficiently be applied to study the temperature dependence of the magnetic anisotropy of thin films. A detailed comparison of the two methods is, however, out of the scope of the present work.

\section{RESULTS AND DISCUSSION}

\section{A. Temperature dependence of the magnetic anisotropy energy}

In order to validate metadynamics for the study of finitetemperature magnetism we first investigated the temperature dependence of the MAE $K(T)$ of a monolayer. According to the theoretical model of Callen and Callen [29] based on on-site anisotropy, the temperature dependence of the MAE is expressed in terms of the magnetization $M(T)$. In the lowtemperature limit the MAE shows a power-law dependence on the magnetization. Although a $K(T) \propto M(T)^{\gamma}$ scaling can be derived for arbitrary temperatures, at elevated temperatures the magnon-magnon interactions, neglected in the theory, become increasingly important; thus, the scaling of the MAE at high temperature will differ from the low-temperature behavior. When $\ell$ is the order of the on-site anisotropy in the spherical harmonics expansion, at low temperature $\gamma=\ell(\ell+$ 1)/2 applies. This implies that for on-site uniaxial anisotropy, i.e., $\ell=2$, the MAE should exhibit a $K(T) \propto M(T)^{3}$ scaling. In the case of strongly itinerant magnetic systems both the on-site and the two-site anisotropy should be present in the spin model, and the scaling behavior of the MAE can be remarkably different from that for only the on-site anisotropy $[12,13]$.

The isotropic exchange couplings in ferromagnetic systems are closely related to the Curie temperatures. For ultrathin films of transition metals the Curie temperature is a few hundred kelvins, and the corresponding effective exchange coupling $J$ is few tens of meV. The uniaxial anisotropy 


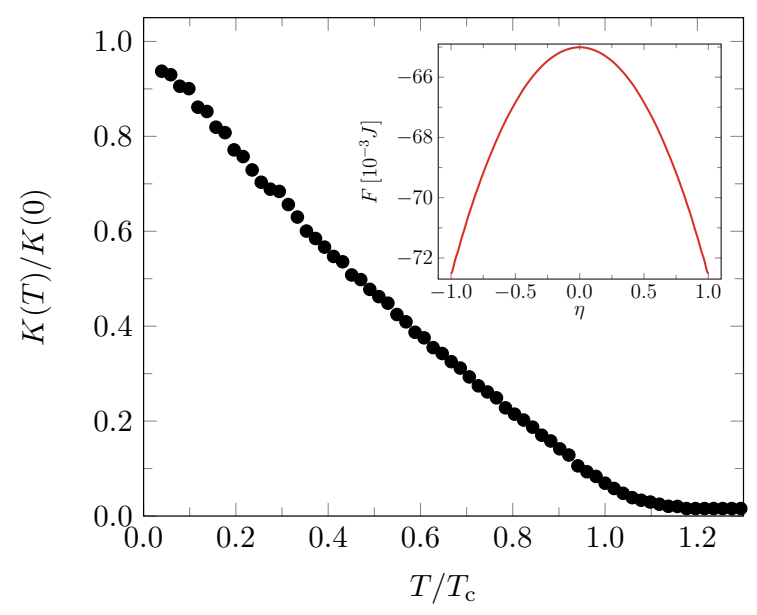

FIG. 1. Temperature dependence of the magnetic anisotropy energy $K(T)$ of a monolayer with uniaxial on-site anisotropy $\lambda=$ $0.01 J$ and vanishing exchange anisotropy $d=0$. In the inset the free energy is shown at $T / T_{\mathrm{C}}=0.2$ as a function of the $\mathrm{CV} \eta$. The simulations have been done on a $32 \times 32$ lattice with the parameters $T_{\mathrm{m}}=10 \mathrm{~J}, w_{0}=0.02 \mathrm{~J}$, and $\sigma=0.03$ (see Sec. II).

constant for hcp Co is $70 \mu \mathrm{eV}$ [30], and for a broad scale of thin films on different substrates it is in the range of 10$200 \mu \mathrm{eV}$ [31]. According to the above experimental values, compared to the effective isotropic coupling, the uniaxial anisotropy constant $(\lambda / J)$ and the anisotropy of the exchange coupling $(d / J)$ have been chosen to be between 0.001 and 0.01 for the subsequent simulations.

The first simulation was performed for a monolayer containing ferromagnetic nearest-neighbor exchange coupling and uniaxial on-site anisotropy with the easy axis perpendicular to the plane $[d=0, \lambda>0$; see Eq. (1)]. The ground state of the system is ferromagnetic with a normal-to-plane orientation. The free energy has a quadratic dependence on the $\mathrm{CV}$, as shown in the inset of Fig. 1. Note that throughout this work the free energy is presented as being normalized to one spin. The parabolic behavior of the free energy is retained in the whole temperature range below the paramagnetic phase transition. The free energy has a maximum at $\eta=0$, referring to the in-plane configuration, and it has minima at $\eta= \pm 1$, representing out-of-plane magnetic orientations. The difference between these two extrema is defined as the MAE. Numerically more efficiently, $K(T)$ can be obtained as the second-order coefficient of a symmetric parabola fitted to the free energy. This is plotted in Fig. 1. As the temperature increases, the curvature of the free energy as a function of CV gradually decreases, and it tends to zero above the Curie temperature. The Curie temperature is identified as the temperature corresponding to the maximum of the specific heat. Although the Curie temperature scales with the system size, it should be chosen to be compatible with the size of the system for which the MAE is calculated.

The magnetic anisotropy in Fig. 1 almost linearly decreases with the temperature, similar to the results obtained by using constrained Monte Carlo simulations [15] for uniaxial anisotropy. The nonzero value of the magnetic anisotropy above the Curie temperature is the consequence of the finite

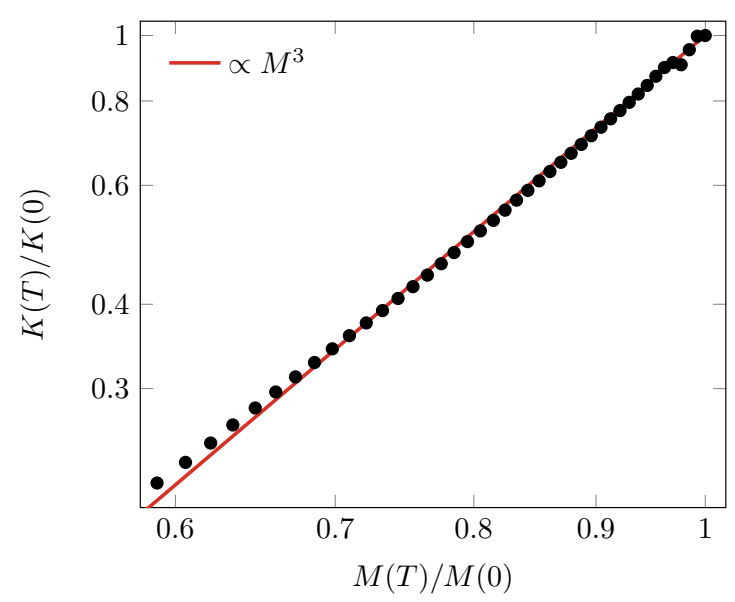

FIG. 2. Log-log plot of the magnetic anisotropy energy of a $32 \times$ 32 square lattice with uniaxial anisotropy $\lambda=0.01 \mathrm{~J}$ and vanishing exchange anisotropy $d=0$ as a function of the magnetization. Note that both the MAE and the magnetization are normalized to zero temperature.

size of the system. In Fig. 2 the MAE is plotted against the magnetization on a log-log mesh. As can be seen, at low temperatures the results show excellent agreement with the scaling behavior predicted by Callen and Callen [29].

If the uniaxial anisotropy $\lambda$ is removed from the model, Eq. (1), and anisotropic exchange $d<0$ is introduced, the scaling behavior of the anisotropy energy will be different, as shown in Fig. 3. At very low temperatures the system behaves as in the case of uniaxial on-site anisotropy, but below $M(T) / M(0) \simeq 0.8$ the exponent $\gamma$ in the relationship $K(T) \propto M(T)^{\gamma}$ changes from 3 to 2 . The occurrence of the exponent $\gamma=2$ in the low-temperature scaling of the MAE was explored in earlier experimental [8] and theoretical studies $[12,13,32]$ for FePt alloys.

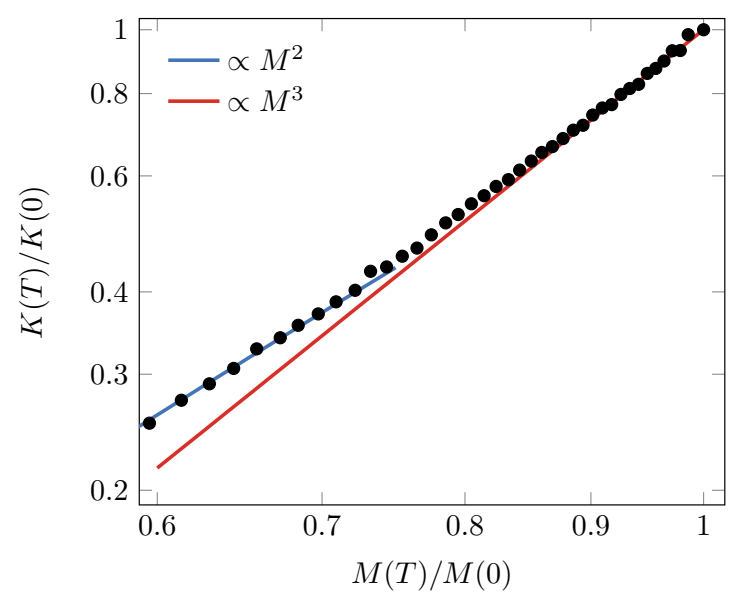

FIG. 3. Log-log plot of the magnetic anisotropy energy of a $32 \times 32$ square lattice with anisotropic exchange coupling $d=0.01 \mathrm{~J}$ and zero on-site anisotropy $\lambda=0$ as a function of the magnetization. Note that both the MAE and the magnetization are normalized to zero temperature. 


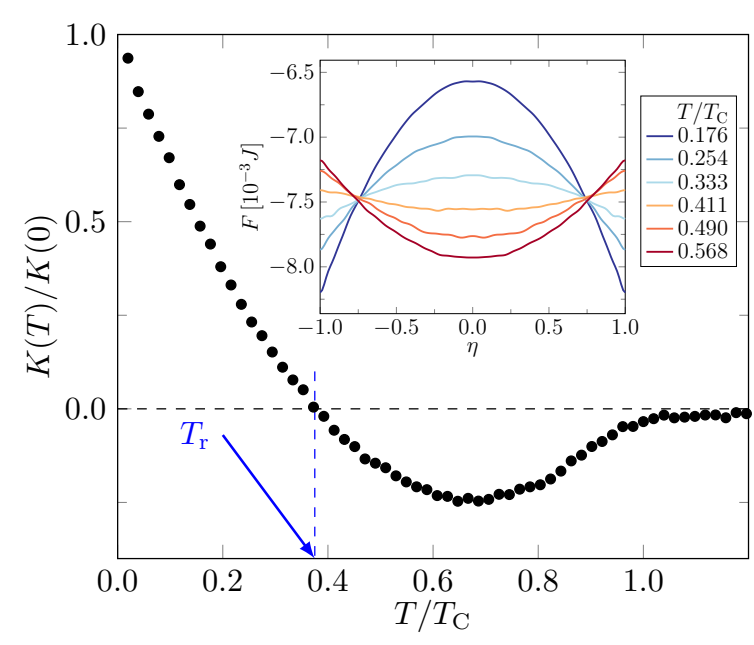

FIG. 4. Magnetic anisotropy energy as normalized to zero temperature of a model monolayer system as a function of the temperature. The metadynamics simulations have been done on a $64 \times 64$ rectangular lattice with competing on-site and nearest-neighbor twosite anisotropy, $\lambda=0.05375 \mathrm{~J}$ and $d=0.025 \mathrm{~J}$, respectively. In the inset the free energy is shown for different temperatures as a function of the collective variable, $\eta=M_{\mathrm{z}} / M$.

\section{B. Spin-reorientation transitions}

The interplay of different types of anisotropies often leads to a reorientation of the magnetization direction. The temperature-driven spin-reorientation transition in thin films is usually explained by the competition of the uniaxial onsite anisotropy and the shape anisotropy [33-36]. For planar systems the shape anisotropy due to the magnetic dipolar interaction always prefers in-plane magnetization, while the on-site anisotropy of a magnetic overlayer frequently prefers a normal-to-plane orientation. The shape anisotropy due to the anisotropic exchange interaction, which is a consequence of the spin-orbit coupling, may also prefer both directions.

In the model given in Eq. (1) the two competing anisotropies are the on-site uniaxial anisotropies $\lambda_{i}$ and the anisotropy of the exchange coupling $d$. Considering a single square lattice, in the case of $\lambda>2 d$ the ground state is a normal-to-plane ferromagnetic. If $\lambda-2 d$ is not too large, a temperature-induced normal-to-plane to in-plane SRT can occur. In the inset of Fig. 4 the free energies for a monolayer with $\lambda=0.05375 \mathrm{~J}$ and $d=0.025 \mathrm{~J}$ are shown for different temperatures, as obtained from metadynamics simulations. At low temperatures the minima of the free energy correspond to $\eta=M_{\mathrm{z}}= \pm 1$, i.e., to a normal-to-plane configuration. As the temperature increases, the curvature of the free energy changes sign, and the minimum of the free energy moves to $\eta=M_{\mathrm{z}}=0$, i.e., to in-plane magnetic orientation. The magnetic anisotropy energy $K(T)$ in Fig. 4 is zero at the transition temperature $T_{\mathrm{r}}$. It is worthwhile to mention that if the magnetization turns into the plane, the system will have a gapless magnetic excitation spectrum, and long-range magnetic order will no longer exist according to the MerminWagner theorem. However, the magnetic anisotropy energy can still be defined as the free-energy difference between the normal-to-plane and in-plane magnetic orientations. The free energy shown in the inset of Fig. 4 demonstrates a first-order phase transition. Moschel and Usadel [37], using MC simulations, and Fridman et al. [38], applying a Hubbard-operator technique, also confirmed that a monolayer exhibits first-order SRT.

In the case of a bilayer our simple model results in a more feature-rich phase diagram where both first-order and second-order SRTs can occur. A mean-field analysis of a very similar model was performed almost two decades ago [39], and here we recall some of the results of that study. As a model system we consider a bilayer on an fcc $(001)$ surface with nearest-neighbor interactions $J$ and $d$ and onsite anisotropy parameters $\lambda_{1}$ and $\lambda_{2}$. At zero temperature, supposing uniform magnetization within each monolayer but different orientations in the two monolayers, the energy of the system can be written as

$$
\begin{aligned}
E= & -4 J+\left(2 d-\lambda_{1}\right) \cos ^{2}\left(\vartheta_{1}\right)+\left(2 d-\lambda_{2}\right) \cos ^{2}\left(\vartheta_{2}\right) \\
& -4 J \cos \left(\vartheta_{1}-\vartheta_{2}\right)+4 d \cos \left(\vartheta_{1}\right) \cos \left(\vartheta_{2}\right),
\end{aligned}
$$

where $\vartheta_{i}$ is the polar angle with respect to the axis perpendicular to the layers $(z)$. In the case of uniform in-plane $\left(\vartheta_{1}=\right.$ $\left.\vartheta_{2}=\pi / 2\right)$ and normal-to-plane $\left(\vartheta_{1}=\vartheta_{2}=0\right)$ orientations the energy has an extremum. The energies of these two particular configurations coincide if $4 d=\lambda_{1}+\lambda_{2}$, defining a line in the $\left\{\lambda_{1}, \lambda_{2}\right\}$ parameter space. In the vicinity of this line a canted magnetic configuration exists. The boundaries of the region of the canted states can be obtained from the stability condition:

$$
\left|\frac{\partial^{2} E}{\partial \vartheta_{i} \vartheta_{j}}\right|_{\vartheta_{i}=0, \pi / 2}=0
$$

yielding the lower boundary line

$$
\left(J+d-\frac{\lambda_{1}^{1}}{2}\right)\left(J+d-\frac{\lambda_{2}^{1}}{2}\right)=(d-J)^{2}
$$

and the upper boundary line

$$
\left(J-2 d+\frac{\lambda_{1}^{\mathrm{u}}}{2}\right)\left(J-2 d+\frac{\lambda_{2}^{\mathrm{u}}}{2}\right)=J^{2} .
$$

Below the line given by Eq. (8), $\lambda_{1}+\lambda_{2}<\lambda_{1}^{1}+\lambda_{2}^{1}$, the ground state is in plane ferromagnetic, and above the line given by Eq. (9), $\lambda_{1}+\lambda_{2}>\lambda_{1}^{\mathrm{u}}+\lambda_{2}^{\mathrm{u}}$, it is normal to the plane ferromagnetic.

At finite temperature the mean-field free energy of the double layer can be expressed as

$$
F=\frac{4 J}{2} \mathbf{M}^{2}-\frac{4 d}{2}\left(M^{z}\right)^{2}-k_{\mathrm{B}} T \ln \left(Z_{1}\right)-k_{\mathrm{B}} T \ln \left(Z_{2}\right),
$$

where

$$
\begin{aligned}
Z_{i}= & \int I_{0}\left[4 J \beta M^{x} \sin (\vartheta)\right] \exp \left[4(J-d) \beta M^{z} \cos (\vartheta)\right] \\
& \times \exp \left[\lambda_{i} \beta \cos ^{2}(\vartheta)\right] \sin (\vartheta) d \vartheta
\end{aligned}
$$

$I_{0}(x)$ is the modified Bessel function of the first kind, and $M_{x}$ and $M_{z}$ are the $x$ and $z$ components of the magnetization of the bilayer, respectively. As was shown in Ref. [39], the magnetization can go to zero either via an in-plane or normal-to-plane direction at temperatures $T_{x}$ and $T_{z}$, respectively, the higher of which can obviously be associated with the mean-field 


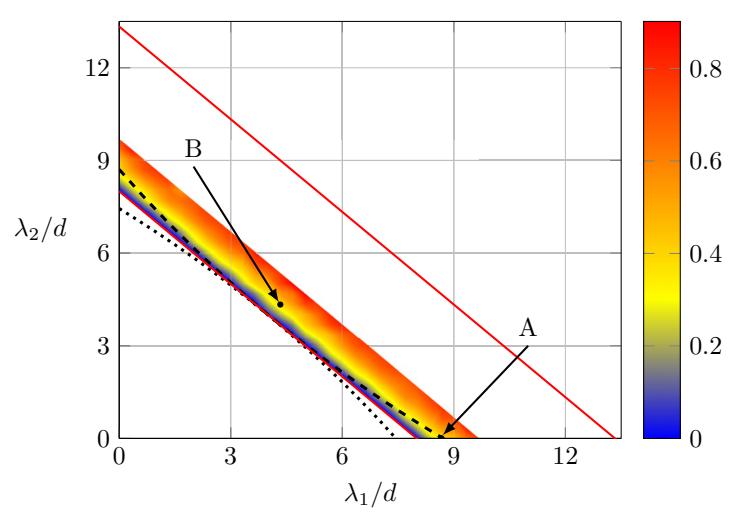

FIG. 5. Phase diagram of an fcc(001) ferromagnetic bilayer described by the model Hamiltonian Eq. (1) with nearest-neighbor exchange interactions, $J$ and $d$, and uniaxial anisotropies, $\lambda_{1}$ and $\lambda_{2}$. For the case of $d=0.005 \mathrm{~J}$, the lower solid red line shows the boundary where the normal-to-plane and in-plane configuration have the same energy, while the region of canted ground states given by Eqs. (8) and (9) is comparable with the linewidth. The upper solid red line bounds the area where a normal-to-plane to in-plane spin reorientation occurs according to mean-field theory. This area becomes considerably narrower from metadynamics simulations, as shown by the colored area. The color bar to the right refers to $T_{\mathrm{r}} / T_{\mathrm{C}}$. The dashed and dotted lines are the boundaries of the region with a canted ground state for $d=0.05 \mathrm{~J}$. For this case, points A $\left(\lambda_{1} / d=8.66, \lambda_{2} / d=0\right)$ and $\mathrm{B}\left(\lambda_{1} / d=\lambda_{2} / d=4.33\right)$ are chosen for further investigations (see the text).

estimation of the Curie temperature $T_{\mathrm{C}}$. Minimizing the free energy with respect to the magnetization of the system with the constraint $M_{z}=0$ or $M_{x}=0$ and using a high-temperature expansion yield the following expressions for $T_{x}$ and $T_{z}$ to first order in $\lambda_{1}$ and $\lambda_{2}$ :

$$
\begin{gathered}
k_{\mathrm{B}} T_{z}=\frac{8}{3}(J-d)+\frac{4}{30}\left(\lambda_{1}+\lambda_{2}\right), \\
k_{\mathrm{B}} T_{x}=\frac{8}{3} J-\frac{2}{30}\left(\lambda_{1}+\lambda_{2}\right) .
\end{gathered}
$$

An out-of-plane to in-plane SRT can occur only when the ground-state magnetization is out of plane and $T_{z}<T_{x}=T_{\mathrm{C}}$. In the case of a reversed SRT the ground-state magnetization has to be in plane (or canted), and $T_{x}<T_{z}=T_{\mathrm{C}}$. In the parameter space $\left\{\lambda_{1}, \lambda_{2}\right\}$ the region where SRT can occur is bounded by the line defined by Eq. (8) and by the line where $T_{x}=T_{z}: \lambda_{1}+\lambda_{2}=\frac{40}{3} d$.

We performed metadynamics Monte Carlo simulations to explore the phase diagram of a model bilayer. Although the anisotropy parameters $\lambda_{i}$ and $d$ can take both positive and negative values, in order to keep the MC simulations tractable, our investigations were restricted to the positive quarter of the parameter space $\left\{\lambda_{1} / d, \lambda_{2} / d\right\}$. The phase diagram for $d=0.005 \mathrm{~J}$ is shown in Fig. 5. In this case, the region where canted ground states exist, determined by Eqs. (8) and (9), is extremely narrow. The area where a normal-toplane to in-plane SRT occurs provided by the metadynamics simulations (colored region) is considerably narrower than the corresponding area predicted by the mean-field theory (bounded by the two solid red lines). The coloring clearly

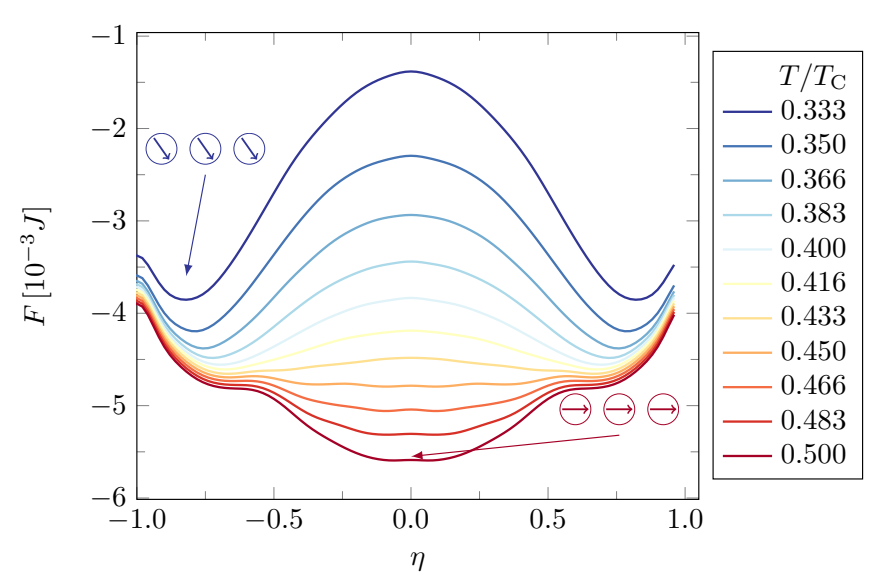

FIG. 6. Free-energy profiles from metadynamics simulations of a $2 \times 64 \times 64$ bilayer with nearest-neighbor exchange interactions, $d=0.05 \mathrm{~J}$, and anisotropy constants $\lambda_{1}=8.66 d, \lambda_{2}=0$ (point A in Fig. 5). The temperature is measured in units of $T_{\mathrm{C}}$. The lowtemperature magnetic configuration is canted $(0<\eta<1)$, while by increasing the temperature the system continuously turns into the phase with in-plane magnetization, showing the nature of a secondorder phase transition.

demonstrates that the reorientation temperature $T_{\mathrm{r}}$ gradually approaches the Curie temperature as the uniaxial anisotropy constants increase, while parallel to the lines $\lambda_{1}+\lambda_{2}=$ const it is almost constant. If the uniaxial anisotropy is further increased, the system keeps its normal-to-plane ferromagnetic order until the ferromagnetic-paramagnetic phase transition.

Increasing the two-site anisotropy $d$, the area of canted ground states on the phase diagram becomes wider. In the case of $d=0.05 \mathrm{~J}$, the lower and upper boundaries of the canted region are indicated by the dotted and dashed lines in Fig. 5, respectively. For further investigations we choose two points in the phase diagram: A $\left(\lambda_{1} / d=8.66, \lambda_{2} / d=0\right)$, representing a canted ground state lying in the vicinity of the upper boundary line of this region (dashed line in Fig. 5), and $\mathrm{B}\left(\lambda_{1} / d=\lambda_{2} / d=4.33\right)$, corresponding to a normalto-plane ferromagnetic ground state. For the first choice of $\left(\lambda_{1}, \lambda_{2}\right)$ the magnetization of the system continuously turns into the plane as the temperature is increased, and considering the normal-to-plane component of the magnetization as the order parameter, the system undergoes a second-order SRT. This is demonstrated in Fig. 6, where the free energies of a $2 \times 64 \times 64$ lattice are shown as a function of the CV at different temperatures close to the SRT. Below the reorientation temperature, $T_{\mathrm{r}} / T_{\mathrm{C}} \sim 0.45$, the magnitude of the minimum position of the free energy $\eta_{\min }$ decreases continuously with increasing temperature, while at the in-plane magnetization $\eta=0$ there is a maximum in the free energy. Above the reorientation transition temperature the in-plane configuration belongs to the minimum of the free energy, which means the order parameter is identical to zero.

If the uniaxial anisotropy parameters are the same for both layers, $\lambda_{1}=\lambda_{2}$, no canted ground state exists for the bilayer; therefore, the mean-field description of temperaturedependent magnetism is analogous to that of the monolayer. The results of metadynamics simulations show, however, 


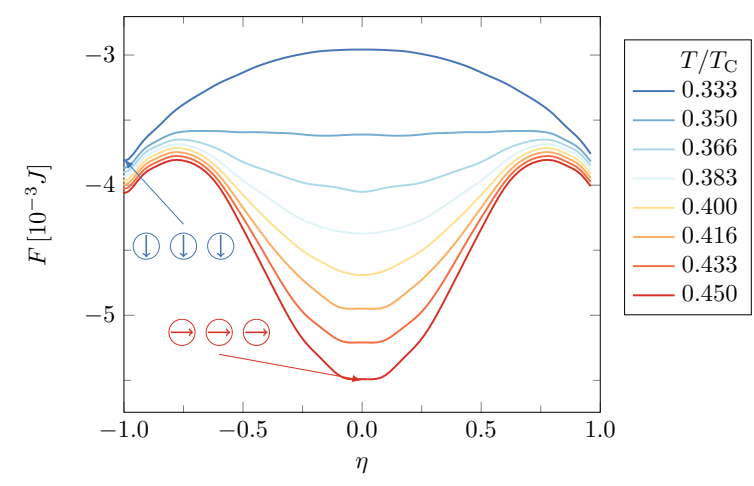

FIG. 7. Free-energy profiles from metadynamics simulations of a $2 \times 64 \times 64$ bilayer with nearest-neighbor exchange interactions, $d=0.05 \mathrm{~J}$, and anisotropy constants $\lambda_{1}=\lambda_{2}=4.33 d$ (point $\mathrm{B}$ in Fig. 5). The temperature is measured in units of $T_{\mathrm{C}}$. At low temperature the magnetization points normal to the plane $(\eta= \pm 1)$, while at the reorientation temperature, $T_{\mathrm{r}} \sim 0.366 T_{\mathrm{C}}$, it suddenly jumps to in plane, as $\eta=0$ becomes the minimum position of the free energy, thus displaying a first-order phase transition.

some different features for the bilayer and the monolayer. According to Fig. 4, the SRT for the monolayer is discontinuous, and the normal-to-plane and in-plane phases cannot coexist. The free energies for the bilayer with anisotropy parameters $\lambda_{1}=\lambda_{2}=4.33 d$ are shown in Fig. 7. Below the reorientation temperature the free energy has minima at $\eta= \pm 1$ which correspond to a normal-to-plane average magnetization. As the temperature increases, a local minimum of the free energy evolves at $\eta=0$, referring to in-plane magnetization. Further increasing the temperature the local maximum at $\eta=0$ becomes the global maximum. The spin-reorientation transition is, therefore, of first order as in the case of the monolayer, but the phases with in-plane and normal-to-plane magnetization can coexist.

\section{C. $\mathrm{Fe}_{2} \mathrm{Au}(001)$}

Over the past three decades, thin iron films deposited on the surface of gold have been the subject of extensive investigations, especially in the context of low-dimensional magnetism (see, e.g., Ref. [40] and references therein). An $\mathrm{Fe}$ monolayer grown on $\mathrm{Au}(001)$ has often been referred to as a prototypical two-dimensional ferromagnet. The film $\mathrm{Fe}_{n} \mathrm{Au}(001)$ exhibits a normal-to-plane magnetic ground state for $n \leqslant 2$, and the magnetisation of the Fe layers undergo a thickness-driven spin reorientation when the thickness of the Fe film reaches three monolayers [40]. While the driving force of this spin reorientation is the magnetostatic shape anisotropy, it is worth studying the temperature dependence of the spin-orbit-induced MAE by using the metadynamics simulations introduced in this work. In this section we present such a study for $\mathrm{Fe}_{2} \mathrm{Au}(001)$.

For the simulations we used the following spin Hamiltonian:

$$
H=-\frac{1}{2} \sum_{p, q=1}^{2} \sum_{i, j} \mathbf{s}_{p i}^{T} \mathcal{J}_{p i, q j} \mathbf{s}_{q j}-\sum_{p=1}^{2} \sum_{i} \lambda_{p}\left(\mathbf{s}_{p i} \hat{\mathbf{z}}\right)^{2},
$$

TABLE I. Calculated layer-dependent magnetic anisotropy parameters (in units of $\mathrm{meV}$ ) for the $\mathrm{Fe}_{2} \mathrm{Au}(001)$ layers. The Fe layer at the interface with $\mathrm{Au}$ is denoted by I, and the one at the surface is denoted by $\mathrm{S}$. Negative (positive) values of the anisotropies prefer the in-plane (normal-to-plane) orientation of the magnetization.

\begin{tabular}{lrr}
\hline \hline Layer & $\lambda$ & $J_{p}^{z z}-J_{p}^{x x}$ \\
\hline $\mathrm{I}$ & -0.097 & 0.181 \\
$\mathrm{~S}$ & 0.360 & -0.314 \\
\hline \hline
\end{tabular}

where $p$ and $q$ denote layers, $i$ and $j$ stand for $\mathrm{Fe}$ atoms within each layer, $\hat{\mathbf{z}}$ is a unit vector parallel to the $z$ axis, $\mathcal{J}_{i j}$ is a $3 \times 3$ matrix of exchange interactions, and the sum in the first term is not restricted to only the nearest neighbors. The trace of the tensor $\mathcal{J}_{i j}$ can be identified as three times the isotropic exchange coupling $J_{i j}$, while the symmetric and antisymmetric parts of the tensor correspond to the pseudodipolar and Dzyaloshinskii-Moriya interactions, respectively [41]. In order to determine the exchange tensors we applied the relativistic extension of the torque method [41] implemented in the framework of the screened Korringa-Kohn-Rostoker (SKKR) method [42]. Since the (001) surface of fcc Au fits almost perfectly on the (001) surface of the bcc Fe (the lattice mismatch is less than $0.6 \%$ ), we used two-dimensional translational symmetry for the whole system using the lattice constant of Au (2.87 $⿱$ A). The Fe-Fe interlayer distance has been chosen to be the same as the bulk value $(1.44 \AA)$, and the $\mathrm{Fe}-\mathrm{Au}$ interlayer distance was $1.6 \AA$.

The calculated spin model parameters were then used in Monte Carlo and metadynamics simulations. In order to reduce finite-size effects, the Curie temperature of the system has been determined from the intersection of the Binder cumulants, yielding $T_{\mathrm{C}} \simeq 380 \mathrm{~K}$, in good agreement with the experiments [43]. In order to characterize the anisotropy of the exchange tensors the lattice sum of the exchange couplings has been introduced:

$$
\mathcal{J}_{p}=\frac{1}{2} \sum_{q=1,2} \sum_{j} \mathcal{J}_{p 0, q j},
$$

where $\mathcal{J}_{p 0, q j}$ is the coupling tensor between an arbitrary site 0 in layer $p$ and site $j$ in the layer $q$. Due to the $C_{4 \mathrm{v}}$ symmetry of the lattice $\mathcal{J}_{p}$ is a diagonal matrix with identical $J_{p}^{x x}$ and $J_{p}^{y y}$ elements.

The layer-dependent uniaxial anisotropy constants $\lambda_{p}$ and the anisotropy of exchange couplings $J_{p}^{z z}-J_{p}^{x x}$ are summarized in Table I. Interestingly, the on-site anisotropies and the exchange anisotropies have different signs in both the interface (I) and surface (S) layers, and they also change sign between the two layers. Nevertheless, in both layers the positive contributions dominate, resulting in an overall normal-to-plane magnetic ground state for the bilayer. The temperature-dependent MAE obtained from metadynamics simulations is plotted in Fig. 8 at low temperatures as a function of the magnetization. It is remarkable that the MAE exhibits a $K \propto M^{3}$ dependence similar to the Callen and Callen theory for $\ell=2$ [29], although the anisotropy has both on-site and two-site contributions (see Table I). We have to 


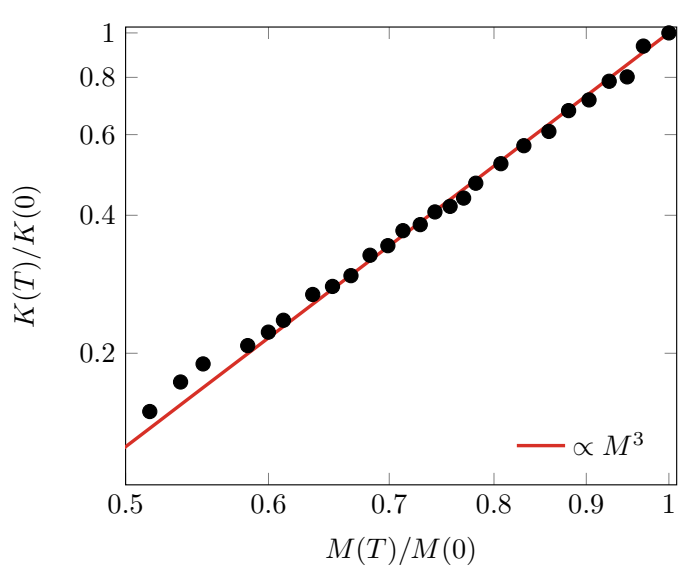

FIG. 8. Temperature dependence of the magnetic anisotropy energy of $\mathrm{Fe}_{2} \mathrm{Au}(001)$. The simulations were performed on a $2 \times$ $64 \times 64$ lattice with the parameters $T_{\mathrm{m}}=10 T_{\mathrm{C}}, w_{0}=0.16 T_{\mathrm{C}}$, and $\sigma=0.04$.

emphasize that in such cases the temperature dependence of the MAE has no predictable power-law form; for systems with uniaxial anisotropy one can expect an exponent between 2 and 3 .

\section{D. $\mathrm{Fe}_{2} \mathrm{~W}(110)$}

Ultrathin Fe films epitaxially grown on W(110) have been studied intensively $[44,45]$ due to their peculiar magnetic properties, such as in- and out-of-plane anisotropy [46], spin reorientation [36,47], and domain wall formation [48]. In this section we consider the double-layer (DL) system $\mathrm{Fe}_{2} \mathrm{~W}(110)$. The magnetic ground state of this system strongly depends on the size and shape of the double-layer areas in the experiments $[36,49]$. Fe DL stripes exhibit a periodic magnetic structure with alternating out-of-plane domains separated by $180^{\circ}$ walls [50]. For larger DL islands there is a normal-to-plane ferromagnetic order at low temperature [47], which turns into the (110) in-plane direction at higher temperature [51].

As for $\mathrm{Fe}_{2} \mathrm{Au}(001)$, the electronic structure of $\mathrm{Fe}_{2} \mathrm{~W}(110)$ was determined self-consistently via the SKKR method, and the relativistic torque method was employed to find the exchange tensors and anisotropy parameters. Since a DL of Fe grows pseudomorphically on W(110) [52], twodimensional translational symmetry is applied throughout the whole system with the lattice constant of bcc bulk W $\left(a_{\mathrm{W}}=3.16 \AA\right)$. According to experimental [53] and theoretical [54] studies, there is a considerable inward relaxation of the $\mathrm{Fe}$ layers due to the large lattice mismatch between $\mathrm{Fe}$ and W. Following Ref. [55], the Fe-W and $\mathrm{Fe}-\mathrm{Fe}$ layer distances were chosen as $2.01 \AA$ and $1.71 \AA$, respectively. In good agreement with previous calculations [48,54], we obtained $2.18 \mu_{\mathrm{B}}$ and $2.73 \mu_{\mathrm{B}}$ for the spinmagnetic moments of $\mathrm{Fe}$ in the surface and interface layers, respectively.

We employed a spin model similar to the one we used for the $\mathrm{Fe}_{2} \mathrm{Au}(001)$ layer, but because of the $C_{2 \mathrm{v}}$ symmetry of the
TABLE II. Calculated layer-dependent magnetic anisotropy parameters (in units of $\mathrm{meV}$ ) for the $\mathrm{Fe}_{2} \mathrm{~W}(110)$ layers. The Fe layer at the interface with $\mathrm{W}$ is denoted by $\mathrm{I}$, and the one at the surface is denoted by $\mathrm{S}$. The notations $x, y$, and $z$ stand for the (1110), (001), and (110) directions, respectively.

\begin{tabular}{lrrrr}
\hline \hline Layer & \multicolumn{1}{c}{$\lambda_{x}$} & \multicolumn{1}{c}{$\lambda_{y}$} & $J_{p}^{z z}-J_{p}^{x x}$ & $J_{p}^{z z}-J_{p}^{y y}$ \\
\hline $\mathrm{I}$ & 0.611 & 0.261 & -0.603 & 0.138 \\
$\mathrm{~S}$ & -0.055 & -0.137 & 0.377 & 0.106 \\
\hline \hline
\end{tabular}

system biaxial anisotropy applies,

$$
\begin{aligned}
H= & -\frac{1}{2} \sum_{p, q=1}^{2} \sum_{i \neq j} \mathbf{s}_{p i}^{T} \mathbf{J}_{p i, q j} \mathbf{s}_{q j} \\
& +\sum_{p=1}^{2} \sum_{i} \lambda_{p x}\left(\mathbf{s}_{p i} \hat{\mathbf{x}}\right)^{2}+\sum_{p=1}^{2} \sum_{i} \lambda_{p y}\left(\mathbf{s}_{p i} \hat{\mathbf{y}}\right)^{2},
\end{aligned}
$$

where $\hat{\mathbf{x}}$ and $\hat{\mathbf{y}}$ are unit vectors parallel to the (1110) and (001) in-plane directions, respectively. The layerwise on-site and exchange anisotropy parameters, as explained in the case of $\mathrm{Fe}_{2} \mathrm{Au}(001)$, are summarized in Table II. The anisotropy of the exchange couplings in the interface layer prefers the in-plane (110) direction which is partially compensated by the contribution from the surface layer. On the contrary, the on-site anisotropy of the interface layer clearly prefers the (110) direction for the magnetization. The MAE calculated as the difference between the energies of the system magnetized in the $(1 \overline{1} 0)$ in-plane direction and parallel to the normal-toplane (110) direction, $E_{1 \overline{1} 0}-E_{110}=0.330 \mathrm{meV}$, as well as the MAE related to the (001) and (110) directions, $E_{001}-$ $E_{110}=0.368 \mathrm{meV}$, imply indeed a normal-to-plane magnetic orientation in the ground state, as also found in Refs. [48,54].

According to susceptibility measurements [51], the Curie temperature strongly depends on the Fe coverage, and in the case of 1.8 monolayers of $\mathrm{Fe} T_{\mathrm{C}}=455 \mathrm{~K}$ was measured. Our simulations on a perfect DL of Fe resulted in a Curie temperature of $520 \mathrm{~K}$, in relatively good agreement with the experiment. In our metadynamics MC simulations the normal-to-plane component of the normalized magnetization was chosen again as the collective variable. In Fig. 9 the magnetic anisotropy energy, defined as the difference of the free energies between the (1 $\overline{1} 0)$ in-plane orientation and the (110) normal-to-plane orientation, is depicted for a wide range below $T_{\mathrm{C}}$. As can be inferred from Fig. 9, the MAE changes sign at $T_{\mathrm{r}}=0.64 T_{\mathrm{C}}$, indicating a SRT from the normal-to-plane to in-plane direction. The driving force of the spin reorientation is most likely a competition between the exchange anisotropy and the on-site anisotropy since these contributions to the MAE exhibit different temperature dependences.

\section{SUMMARY}

We introduced metadynamics combined with Monte Carlo simulations to study the thermal equilibrium of magnetic systems and demonstrated that the method can be applied to the temperature dependence of magnetic anisotropy of thin 


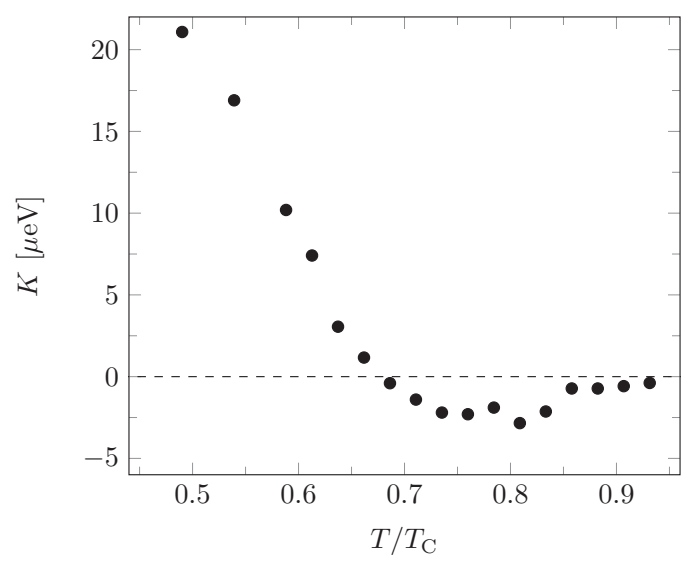

FIG. 9. Calculated magnetic anisotropy energy, $K(T)=$ $F_{1 \overline{1} 0}(T)-F_{110}(T)$, of an Fe DL on top of W(110) as obtained by well-tempered metadynamics MC simulations on a $2 \times 64 \times 64 \times$ lattice with the parameters $T_{\mathrm{m}}=2 T_{\mathrm{C}}, w_{0}=0.02 T_{\mathrm{C}}$, and $\sigma=0.04$.

films. In particular, we reproduced the power-law scaling of the magnetic anisotropy vs magnetization proposed by Callen and Callen [29] as for systems with on-site uniaxial anisotropy the simulations provided an exponent of 3, whereas in the case of dominating exchange anisotropy an exponent of 2 was obtained in the high-temperature regime.

We applied the method to explore spin-reorientation transitions in thin films. By using a simple spin model, first, we performed a detailed analysis of the SRT for a monolayer and a double layer. For double layers we showed that, by setting appropriate model parameters, both first- and secondorder SRTs can occur, as predicted within the mean-field theory. Then we considered two kinds of iron double-layer systems with perpendicular magnetic anisotropy where we set up a more complex spin model containing tensorial exchange interactions calculated from first-principles methods. In the case of $\mathrm{Fe}_{2} \mathrm{Au}(001)$ the MAE followed the usual $M^{3}$ power law, and no SRT was observed. In the case of $\mathrm{Fe}_{2} \mathrm{~W}(110)$ the MAE showed a more complex temperature dependence, and our simulations reproduced the normal-to-plane to in-plane SRT seen in experiments [36,47].

One of the future challenges for the simulations based on $a b$ initio spin models is posed by exploring the effect of the Dzyaloshinskii-Moriya interactions on the temperature dependence of the magnetic anisotropy in thin films proposed recently [56]. Moreover, the calculated temperature-dependent magnetic anisotropy energies can be used to estimate the transverse susceptibility needed as input for large-scale micromagnetic simulations based on the Landau-Lifshitz-Bloch equation [57].

\section{ACKNOWLEDGMENTS}

The authors are grateful for the financial support from the National Research, Development and Innovation Office of Hungary under Project No. K115575, as well as from the BME Nanotechnology FIKP grant (BME FIKP-NAT).
[1] L. Néel, J. Phys. Radium 15, 225 (1954).

[2] S. Iwasaki, Proc. Jpn. Acad., Ser. B 85, 37 (2009).

[3] B. Tudu and A. Tiwari, Vacuum 146, 329 (2017).

[4] B. Dieny and M. Chshiev, Rev. Mod. Phys. 89, 025008 (2017).

[5] H. F. Hamann, Y. C. Martin, and H. K. Wickramasinghe, Appl. Phys. Lett. 84, 810 (2004).

[6] T. W. McDaniel, J. Phys.: Condens. Matter 17, R315 (2005).

[7] B. C. Stipe, T. C. Strand, C. C. Poon, H. Balamane, T. D. Boone, J. A. Katine, J.-L. Li, V. Rawat, H. Nemoto, A. Hirotsune, O. Hellwig, R. Ruiz, E. Dobisz, D. S. Kercher, N. Robertson, T. R. Albrecht, and B. D. Terris, Nat. Photonics 4, 484 (2010).

[8] S. Okamoto, N. Kikuchi, O. Kitakami, T. Miyazaki, Y. Shimada, and K. Fukamichi, Phys. Rev. B 66, 024413 (2002).

[9] J.-U. Thiele, K. R. Coffey, M. F. Toney, J. A. Hedstrom, and A. J. Kellock, J. Appl. Phys. 91, 6595 (2002).

[10] C. A. F. Vaz, J. A. C. Bland, and G. Lauhoff, Rep. Prog. Phys. 71, 056501 (2008).

[11] Y. Fu, I. Barsukov, J. Li, A. M. Gonçalves, C. C. Kuo, M. Farle, and I. N. Krivorotov, Appl. Phys. Lett. 108, 142403 (2016).

[12] J. B. Staunton, S. Ostanin, S. S. A. Razee, B. L. Gyorffy, L. Szunyogh, B. Ginatempo, and E. Bruno, Phys. Rev. Lett. 93, 257204 (2004).

[13] O. N. Mryasov, U. Nowak, K. Y. Guslienko, and R. W. Chantrell, Europhys. Lett. 69, 805 (2005).

[14] A. Buruzs, P. Weinberger, L. Szunyogh, L. Udvardi, P. I. Chleboun, A. M. Fischer, and J. B. Staunton, Phys. Rev. B 76, 064417 (2007).
[15] P. Asselin, R. F. L. Evans, J. Barker, R. W. Chantrell, R. Yanes, O. Chubykalo-Fesenko, D. Hinzke, and U. Nowak, Phys. Rev. B 82, 054415 (2010).

[16] F. Wang and D. P. Landau, Phys. Rev. Lett. 86, 2050 (2001).

[17] S. Marsili, A. Barducci, R. Chelli, P. Procacci, and V. Schettino, J. Chem. Phys. B 110, 14011 (2006).

[18] A. Laio and M. Parrinello, Proc. Natl. Acad. Sci. USA 99, 12562 (2002).

[19] A. Barducci, G. Bussi, and M. Parrinello, Phys. Rev. Lett. 100, 020603 (2008).

[20] J. F. Dama, M. Parrinello, and G. A. Voth, Phys. Rev. Lett. 112, 240602 (2014).

[21] J. Tóbik, R. Martoňák, and V. Cambel, Phys. Rev. B 96, 140413(R) (2017).

[22] U. Nowak, Classical spin models, in Handbook of Magnetism and Advanced Magnetic Materials, edited by H. Kronmüller and S. Parkin (John Wiley and Sons, New York, 2007).

[23] F. Marini, C. Camilloni, D. Provasi, R. Broglia, and G. Tiana, Gene 422, 37 (2008).

[24] Y. Crespo, F. Marinelli, F. Pietrucci, and A. Laio, Phys. Rev. E 81, 055701(R) (2010).

[25] N. Metropolis, A. W. Rosenbluth, M. N. Rosenbluth, A. H. Teller, and E. Teller, J. Chem. Phys. 21, 1087 (1953).

[26] A. Laio and F. L. Gervasio, Rep. Prog. Phys. 71, 126601 (2008).

[27] P. Raiteri, A. Laio, F. L. Gervasio, C. Micheletti, and M. Parrinello, J. Phys. Chem. B 110, 3533 (2006).

[28] G. Torrie and J. Valleau, J. Comput. Phys. 23, 187 (1977). 
[29] H. Callen and E. Callen, J. Phys. Chem. Solids 27, 1271 (1966).

[30] K. Baberschke, in Band-Ferromagnetism: Ground-State and Finite-Temperature Phenomena, edited by K. Baberschke, W. Nolting, and M. Donath (Springer, Berlin, 2001), pp. 27-45.

[31] M. T. Johnson, P. J. H. Bloemen, F. J. A. den Broeder, and J. J. de Vries, Rep. Prog. Phys. 59, 1409 (1996).

[32] A. Deák, E. Simon, L. Balogh, L. Szunyogh, M. dos Santos Dias, and J. B. Staunton, Phys. Rev. B 89, 224401 (2014).

[33] D. P. Pappas, K.-P. Kämper, and H. Hopster, Phys. Rev. Lett. 64, 3179 (1990).

[34] O. Fruchart, J.-P. Nozières, and D. Givord, J. Magn. Magn. Mater. 165, 508 (1997).

[35] R. Sellmann, H. Fritzsche, H. Maletta, V. Leiner, and R. Siebrecht, Phys. B (Amsterdam, Neth.) 276-278, 578 (2000).

[36] T. Slezak, M. Slezak, M. Zajac, K. Freindl, A. Kozioł-Rachwał, K. Matlak, N. Spiridis, D. Wilgocka-Slezak, E. PartykaJankowska, M. Rennhofer, A. I. Chumakov, S. Stankov, R. Rüffer, and J. Korecki, Phys. Rev. Lett. 105, 027206 (2010).

[37] A. Moschel and K. D. Usadel, Phys. Rev. B 51, 16111 (1995).

[38] Yu. A. Fridman, D. V. Spirin, and Ph. N. Klevets, J. Magn. Magn. Mater. 253, 105 (2002).

[39] L. Udvardi, L. Szunyogh, A. Vernes, and P. Weinberger, Philos. Mag. B 81, 613 (2001).

[40] D. Wilgocka-Slezak, K. Freindl, A. Kozioł, K. Matlak, M. Rams, N. Spiridis, M. Slezak, T. Slezak, M. Zajac, and J. Korecki, Phys. Rev. B 81, 064421 (2010).

[41] L. Udvardi, L. Szunyogh, K. Palotás, and P. Weinberger, Phys. Rev. B 68, 104436 (2003).
[42] L. Szunyogh, B. Újfalussy, and P. Weinberger, Phys. Rev. B 51, 9552 (1995).

[43] W. Dürr, M. Taborelli, O. Paul, R. Germar, W. Gudat, D. Pescia, and M. Landolt, Phys. Rev. Lett. 62, 206 (1989).

[44] D. Sander, Rep. Prog. Phys. 62, 809 (1999).

[45] H. L. Meyerheim, D. Sander, R. Popescu, J. Kirschner, P. Steadman, and S. Ferrer, Phys. Rev. B 64, 045414 (2001).

[46] J. Hauschild, U. Gradmann, and H. J. Elmers, Appl. Phys. Lett. 72, 3211 (1998).

[47] N. Weber, K. Wagner, H. J. Elmers, J. Hauschild, and U. Gradmann, Phys. Rev. B 55, 14121 (1997).

[48] M. Heide, G. Bihlmayer, and S. Blügel, Phys. Rev. B 78, 140403(R) (2008).

[49] K. von Bergmann, M. Bode, and R. Wiesendanger, J. Magn. Magn. Mater. 305, 279 (2006).

[50] H. J. Elmers, J. Hauschild, and U. Gradmann, Phys. Rev. B 59, 3688 (1999).

[51] M. J. Dunlavy and D. Venus, Phys. Rev. B 69, 094411 (2004).

[52] U. Gradmann and G. Waller, Surface Sci. 116, 539 (1982).

[53] B. Santos, M. Rybicki, I. Zasada, E. Starodub, K. F. McCarty, J. I. Cerda, J. M. Puerta, and J. de la Figuera, Phys. Rev. B 93, 195423 (2016).

[54] X. Qian and W. Hübner, Phys. Rev. B 60, 16192 (1999).

[55] M. Heide, Ph.D. thesis, Rheinisch-Westfälische Technische Hochschule Aachen, 2006.

[56] L. Rózsa, U. Atxitia, and U. Nowak, Phys. Rev. B 96, 094436 (2017).

[57] U. Atxitia, D. Hinzke, and U. Nowak, J. Phys. D 50, 033003 (2016). 